\title{
An Improved Peak Sidelobe Reduction Method for Subarrayed Beam Scanning
}

\author{
Hang Hu, ${ }^{1}$ Xuepeng Luan, ${ }^{2}$ Guanglei Zhang, ${ }^{3}$ Ke Song, ${ }^{4}$ Weihui Liu, ${ }^{1}$ and Chenghu Mou ${ }^{4}$ \\ ${ }^{1}$ School of Electronics and Information Engineering, Harbin Institute of Technology, Harbin 150001, China \\ ${ }^{2}$ College of Computer \& Information Science, Three Gorges University, Yichang, Hubei 443002, China \\ ${ }^{3}$ RAA Integrated Microwave RF Simulation System, Radar and Avionics Institute of AVIC, Wuxi, Jiangsu 214063, China \\ ${ }^{4}$ No. 802 Institute of SAST, Shanghai 200090, China
}

Correspondence should be addressed to Hang Hu; huhang@hit.edu.cn

Received 20 June 2014; Accepted 6 December 2014

Academic Editor: Ahmed Shaharyar Khwaja

Copyright (C) 2015 Hang Hu et al. This is an open access article distributed under the Creative Commons Attribution License, which permits unrestricted use, distribution, and reproduction in any medium, provided the original work is properly cited.

\begin{abstract}
This paper focused on PSL (peak sidelobe level) reduction for subarrayed beam scanning in phased array radars. The desired GSP (Gaussian Subarray Patterns) are achieved by creating a subarray weighting network. The GSP-based method could reduce PSL of array pattern; compared with the method based on the desired subarray pattern which is defined by ideal space-domain filter, the PSL reduction performance is improved remarkably. Further, based on the concept adopting superelement patterns to approximately express original subarray patterns, the simplified GSP-based method is proposed. So the dimension of each matrix required for creating the weighting network, which was originally the same as the element number, could be reduced to the same as the subarray number. Consequently, we achieve remarkable reduction of the computation burden; simultaneously, the PSL mitigation performance is degraded slightly. Simulation results demonstrate the validity of the introduced methods.
\end{abstract}

\section{Introduction}

In modern PAR (phased array radars) and particularly multifunctional PAR, the subarray structure is usually adopted [1$4]$ and subarrayed array processing is a key technique [5-9]. For subarrayed PAR, the look direction is usually controlled by the phase shifters [1]. However, further digital scanning at subarray level is required for forming multiple beams and many other applications. The look direction is steered into a certain direction by the phase shifters and then SBS (subarrayed beam scanning) is used for a limited sector of look directions around the presteered direction.

Typically with SBS, the PSL will increase rapidly with the scanning direction departing from the original look direction. Therefore, the requirement for PSL reduction arises. The resulting array pattern is composed of the pattern of each subarray. Usually, the shape and beamwidth of the subarray patterns are different. Each subarray pattern has a high PSL which contributes to a high PSL of the array pattern. If the pattern of each subarray would have good coherence in the main beam and have a low PSL, the PSL of the array pattern could be reduced for SBS.

Nickel made a first investigation for reducing the PSL with SBS. In his paper [4], the SLL with SBS was reduced to some degree by inserting a matrix operation with the objective of making the new subarray pattern approach an ideal one based on LMS criterion. Therein, the ideal subarray pattern is set as Ideal Space-Domain Filter (ISDF in short) which is constant within the main lobe and zero otherwise.

This paper introduces Gaussian Subarray Pattern (GSP for short) [5] as the desired pattern in order to improve the PSL with SBS. For the GSP, the gain is set very low outside the main beam which is better suited to reduce the PSL. However, in GSP-based method, the dimension of the matrix for creating WN (weighting network) is the same as the element number. Therefore, the computational burden is enormous for the PAR containing hundreds or even thousands of elements. 
Therefore, this paper adopts the methodology of regarding the array as a superarray and each subarray as a superelement, and representing the superelements by the corresponding subarray's phase center and gain. When creating the $\mathrm{WN}$, the superelement pattern approximately represents the pattern for the new subarray obtained by WN. By using this simplification, the dimension of each matrix for creating $\mathrm{WN}$ would be reduced to the subarray number. Therefore, the simplification could reduce the computational burden remarkably, compared with GSP-based one. This simplification method is named Simplified GSP (SGSP for short) in this paper.

\section{Array Pattern of SBS}

Assuming a planar phased array with $M$ omnidirectional elements located on the xoy plane, take the first element as the origin of the coordinate system and let $\left(x_{m}, y_{m}\right)$ be the coordinate of the $m$ th $(m=1, \ldots, M)$ element. Amplitude weighting at element is used for reducing the PSL of the sum beam. The weight of the $m$ th element is $w_{\text {ele_m }}$ and array look direction is $\left(\theta_{0}, \varphi_{0}\right)$.

Let the phased array be divided into $L$ subarrays, and let $\mathbf{T}_{0}$ represent the subarray forming matrix of $M \times$ $L$ dimension. Let $\mathbf{W}_{\text {ele }}=\operatorname{diag}\left(w_{\text {ele } \_m}\right)_{m=1,2, \ldots, M}$; denote $\varphi_{m}(\theta, \varphi)=\exp \left\{-j 2 \pi\left[x_{m} \sin \theta \cos \varphi+y_{m} \sin \theta \sin \varphi\right] / \lambda\right\}$ and $\Phi_{0}=\operatorname{diag}\left[\varphi_{m}^{*}\left(\theta_{0}, \varphi_{0}\right)\right]_{m=1, \ldots, M}$; then, subarray transformation matrix is

$$
\mathbf{T}=\Phi_{0} \mathbf{W}_{\mathrm{ele}} \mathbf{T}_{0}
$$

We consider the array as a superarray and each subarray as a superelement. The superelement position is defined as the phase centre of the subarray. For the $l$ th subarray, the phase center is denoted by $\xi_{l, x}$ and $\xi_{l, y}$ along the $x$ and $y$ direction, respectively, and the gain is denoted by $g_{l}$. Then, one obtains [5]

$$
\begin{gathered}
\xi_{l, p}=\frac{\sum_{m \in U_{l}} w_{\text {ele } \_m} p_{m}}{\sum_{m \in U_{l}} w_{\text {ele } \_m}}, \text { for } p=x \text { or } y, \\
g_{l}=\sum_{m \in U_{l}} w_{\text {ele } \_m},
\end{gathered}
$$

where $U_{l}$ denotes the set of indices of elements in the $l$ th subarray.

We introduce $a_{m}(u, v)=e^{j 2 \pi\left(x_{m} u+y_{m} v\right) / \lambda}$ and define $\mathbf{a}(u, v)=\left[a_{1}(u, v), \ldots, a_{m}(u, v), \ldots, a_{M}(u, v)\right]^{\mathrm{T}}$. Suppose that $f_{l}(u, v)$ is the pattern of the $l$ th subarray and we let $\mathbf{f}_{\text {sub }}(u, v)=$ $\left[f_{1}(u, v), \ldots, f_{l}(u, v), \ldots, f_{L}(u, v)\right]^{\mathrm{T}}$; then,

$$
\mathbf{f}_{\text {sub }}(u, v)=\mathbf{T}^{\mathrm{H}} \mathbf{a}(u, v) .
$$

The array pattern is

$$
F(u, v)=\sum_{l=1}^{L} f_{l}(u, v) e^{j 2 \pi\left(\xi_{l, x} u+\xi_{l, y} v\right)}
$$

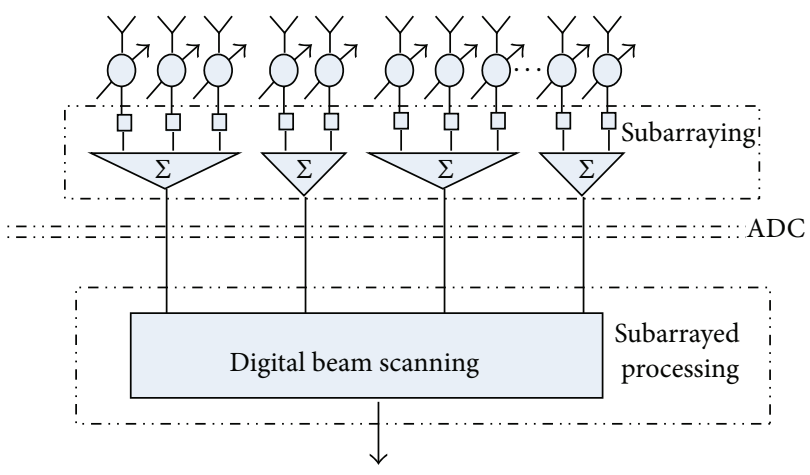

FiguRE 1: Structure of subarrayed beam scanning.

SBS is implemented by performing subarrayed digital phase shift while taking a superelement as reference element. The array pattern after scanning in this way is

$$
F_{\text {Scan }}(u, v)=\sum_{l=1}^{L} f_{l}(u, v) e^{j 2 \pi\left[\xi_{l, x}(u+\Delta u)+\xi_{l, y}(v+\Delta v)\right]}
$$

in which $\Delta u$ and $\Delta v$ represent the scan angles in $u$ and $v$ direction.

The concept of the subarrayed beam scanning is indicated in Figure 1.

\section{SGSP-Based SLL Reduction for SBS}

To suppress the PSL after SBS, a way is postprocessing the subarray outputs, which creates new subarrays with new patterns and new superelement. This is designed to make the new subarray pattern approximate to a desired subarray pattern according to the LMS criterion. The starting point of creating the $\mathrm{WN}$ is to make the new subarrays have a pattern with similar shape within the main beam and with PSLs as low as possible.

3.1. GSP-Based Weighting Network. Firstly, the desired array pattern is set as one with a shape of a Gaussian function. For lth subarray, it is expressed as [5]

$$
\begin{aligned}
f_{l}^{(\mathrm{GSP})}(u, v)= & \frac{1}{\pi \sigma^{2}} e^{-\left[\left(u-u_{0}\right)^{2}+\left(v-v_{0}\right)^{2}\right] / \sigma^{2}} \\
& \cdot e^{j 2 \pi\left[\xi_{l, x}\left(u-u_{0}\right)+\xi_{l, y}\left(v-v_{0}\right)\right] / \lambda},
\end{aligned}
$$

where $\sigma^{2}$ represents the variance of Gaussian distribution function which is used to adjust the beamwidth.

The WN $\mathbf{W}=\left(\mathbf{w}_{1}, \ldots, \mathbf{w}_{l}, \ldots, \mathbf{w}_{L}\right)$ in which $\mathbf{w}_{l}$ is an $L$-dimensional column vector. The pattern of the $l$ th new subarray is

$$
f_{\text {new } l}(u, v)=\left(\mathbf{w}_{l}\right)^{\mathrm{H}} \mathbf{f}_{\text {sub }}(u, v) .
$$

We consider the objective function

$$
Y_{l}=\int_{V}\left|f_{\text {new } \perp}(u, v)-f_{l}^{(\mathrm{GSP})}(u, v)\right|^{2} \mathrm{~d} u \mathrm{~d} v,
$$

where $V=\left\{(u, v) \in \mathbb{R}^{2} \mid u^{2}+v^{2} \leq 1\right\}$. Then, we have [5]

$$
\mathbf{W}=\left(\mathbf{T}^{\mathrm{H}} \mathbf{T}\right)^{-1} \mathbf{T}^{\mathrm{H}} \mathbf{C}^{-1} \mathbf{V} \text {, }
$$


where

$$
\mathbf{C}=\int_{V} \mathbf{a}(u, v) \mathbf{a}^{\mathrm{H}}(u, v) \mathrm{d} u \mathrm{~d} v
$$

and $\mathbf{V}=\left(\mathbf{v}_{1}, \ldots, \mathbf{v}_{l}, \ldots, \mathbf{v}_{L}\right)$, where $\mathbf{v}_{l}=\left(v_{1, l}, \ldots, v_{m, l}, \ldots\right.$, $\left.v_{M, l}\right)^{\mathrm{T}}$ and

$$
\begin{aligned}
v_{m, l}= & \frac{1}{\pi \sigma^{2}} e^{j 2 \pi\left(x_{m} u_{0}+y_{m} v_{0}\right) / \lambda} \\
& \cdot \int_{V} e^{j 2 \pi\left[\left(x_{m}-\xi_{l, x}\right)\left(u-u_{0}\right)+\left(y_{m}-\xi_{l, y}\right)\left(v-v_{0}\right)\right] / \lambda} \\
& \cdot e^{-\left[\left(u-u_{0}\right)^{2}+\left(v-v_{0}\right)^{2}\right] / \sigma^{2}} \mathrm{~d} u \mathrm{~d} v, \quad 1 \leq m \leq M .
\end{aligned}
$$

Considering the formula (9), T, C, and $\mathbf{V}$ have the dimensions $M \times L, M \times M$, and $M \times L$, respectively. Usually, $M$ has an order of magnitude from several hundred to several thousand, so their extensive computations are needed to calculate W.

3.2. SGSP-Based Weighting Network. A method of reducing the computational cost is proposed in the following. The idea is to represent the pattern function of the new subarray in (7) approximately. We use $\mathbf{A}_{\mathrm{se}}\left(u_{0}, v_{0}\right)$ denoting the steering vector matrix of the superarray in the look direction:

$$
\mathbf{A}_{\text {se }}\left(u_{0}, v_{0}\right)=\operatorname{diag}\left(e^{j 2 \pi\left(\xi_{l, x} u_{0}+\xi_{l, y} v_{0}\right) / \lambda}\right)_{l=1, \ldots, L} .
$$

And let

$$
\mathbf{G}_{\mathrm{se}}=\operatorname{diag}\left(g_{l}\right)_{l=1, \ldots, L} .
$$

Phase shifts and gains are combined into

$$
\mathbf{B}_{\mathrm{se}}=\mathbf{A}_{\mathrm{se}}^{\mathrm{H}}\left(u_{0}, v_{0}\right) \mathbf{G}_{\mathrm{se}} \cdot
$$

The steering vector of superarray is expressed as

$$
\mathbf{a}_{\mathrm{se}}(u, v)=\left[a_{\mathrm{se} \_}(u, v), \ldots, a_{\mathrm{se} \perp}(u, v), \ldots, a_{\mathrm{se} \perp L}(u, v)\right]^{\mathrm{T}},
$$

where $a_{\text {se }\lrcorner}(u, v)=e^{j 2 \pi\left(\xi_{l, x} u+\xi_{l, y} v\right) / \lambda}$.

Let

$$
\mathbf{f}_{\text {sub }}^{\prime}(u, v)=\mathbf{B}_{\mathrm{se}} \mathbf{a}_{\mathrm{se}}(u, v)
$$

and denote $\mathbf{f}_{\text {sub }}^{\prime}(u, v)=\left[f_{1}^{\prime}(u, v), \ldots, f_{l}^{\prime}(u, v), \ldots, f_{L}^{\prime}(u, v)\right]^{\mathrm{T}}$. Substituting (14) and (15) into (16), one obtains

$$
f_{l}^{\prime}(u, v)=g_{l} e^{j 2 \pi\left[\xi_{l, x}\left(u-u_{0}\right)+\xi_{l, y}\left(v-v_{0}\right)\right] / \lambda} .
$$

So $\mathbf{f}_{\text {sub }}^{\prime}(u, v)$ is used to express the subarray pattern approximately.

Next, we adopt the $\mathrm{WN} \mathrm{W}^{\prime}$ and denote it as $\mathbf{W}^{\prime}=\left(\mathbf{w}_{1}^{\prime}, \ldots\right.$, $\left.\mathbf{w}_{l}^{\prime}, \ldots, \mathbf{w}_{L}^{\prime}\right)$. The pattern of the new subarray obtained by $\mathbf{W}^{\prime}$ is

$$
f_{\text {new } \_l}^{\prime}(u, v)=\left(\mathbf{w}_{l}^{\prime}\right)^{H} \mathbf{f}_{\text {sub }}^{\prime}(u, v) \text {. }
$$

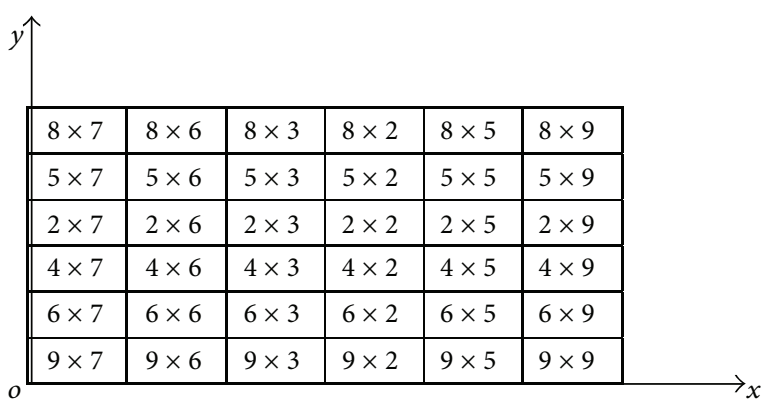

Figure 2: Antenna with 1088 elements on rectangular grid configured into 36 subarrays.

We determine the subarray weight such that a desired pattern is approximated in a LMS sense:

$$
Y_{l}^{\prime}=\int_{V}\left|f_{\text {new } \perp}^{\prime}(u, v)-f_{l}^{(G S P)}(u, v)\right|^{2} \mathrm{~d} u \mathrm{~d} v .
$$

Similarly, we can obtain [5]

$$
\mathbf{W}^{\prime}=\left(\mathbf{B}_{\mathrm{se}} \mathbf{B}_{\mathrm{se}}^{\mathrm{H}}\right)^{-1} \mathbf{B}_{\mathrm{se}}\left(\mathbf{C}_{\mathrm{se}}\right)^{-1} \mathbf{V}_{\mathrm{se}},
$$

where

$$
\mathbf{C}_{\mathrm{se}}=\int_{V} \mathbf{a}_{\mathrm{se}}(u, v) \mathbf{a}_{\mathrm{se}}^{\mathrm{H}}(u, v) \mathrm{d} u \mathrm{~d} v
$$

and $\mathbf{V}_{\text {se }}=\left(\mathbf{v}_{1}^{\prime}, \ldots, \mathbf{v}_{l}^{\prime}, \ldots, \mathbf{v}_{L}^{\prime}\right)$, where $\mathbf{v}_{l}^{\prime}=\left(v_{1, l}^{\prime}, \ldots, v_{n, l}^{\prime}\right.$, $\left.\ldots, v_{L, l}^{\prime}\right)^{\mathrm{T}}$ and

$$
\begin{aligned}
v_{n, l}^{\prime}= & \frac{1}{\pi \sigma^{2}} e^{j 2 \pi\left(\xi_{n, x} u_{0}+\xi_{n, y} v_{0}\right) / \lambda} \\
& \cdot \int_{V} e^{j 2 \pi\left[\left(\xi_{n, x}-\xi_{h, x}\right)\left(u-u_{0}\right)+\left(\xi_{n, y}-\xi_{h, y}\right)\left(v-v_{0}\right)\right] / \lambda} \\
& \cdot e^{-\left[\left(u-u_{0}\right)^{2}+\left(v-v_{0}\right)^{2}\right] / \sigma^{2}} \mathrm{~d} u \mathrm{~d} v, \quad 1 \leq n \leq L .
\end{aligned}
$$

Considering formula (20), the matrices required in creating $\mathbf{W}^{\prime}$, that is, $\mathbf{V}_{\mathrm{se}}, \mathbf{B}_{\mathrm{se}}$, and $\mathbf{C}_{\mathrm{se}}$, are all of dimension $L \times L$. Usually $L$ is only a few dozens of magnitude and therefore the computational burden with $\mathbf{W}^{\prime}$ is reduced remarkably compared with $\mathbf{W}$.

\section{Simulation Results}

Assume a planar array with $32 \times 34$ omnidirectional elements placed at rectangle grid on xoy plane, and the spacing between adjacent elements is $\lambda / 2$. A $-40 \mathrm{~dB}$ Taylor weighting is applied in both $x$ and $y$ directions. The array is divided into $6 \times 6$ subarrays and each of which is a rectangular array. The element distribution and the subarray arrangement are shown in Figure 2. We assume that the look direction is $(-0.5$, 0 ), $\mathrm{BW}$ is the $3 \mathrm{~dB}$ beam width of the pattern, and $\sigma^{2}$ is chosen as $0.2 \mathrm{BW}$ in both the GSP and the SGSP cases.

4.1. Subarray Patterns. Figure 3 shows subarray patterns obtained by different methods. Figure 3(a) shows the original 


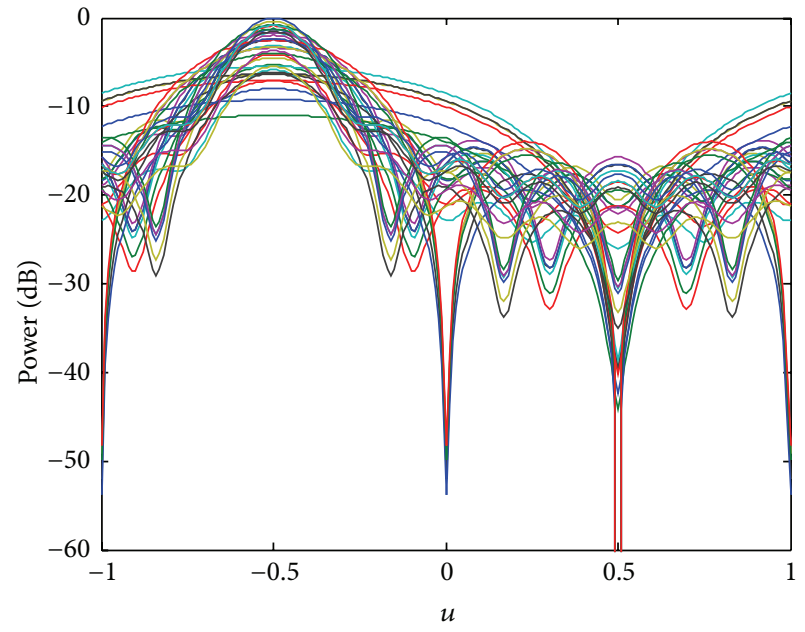

Azimuth cut

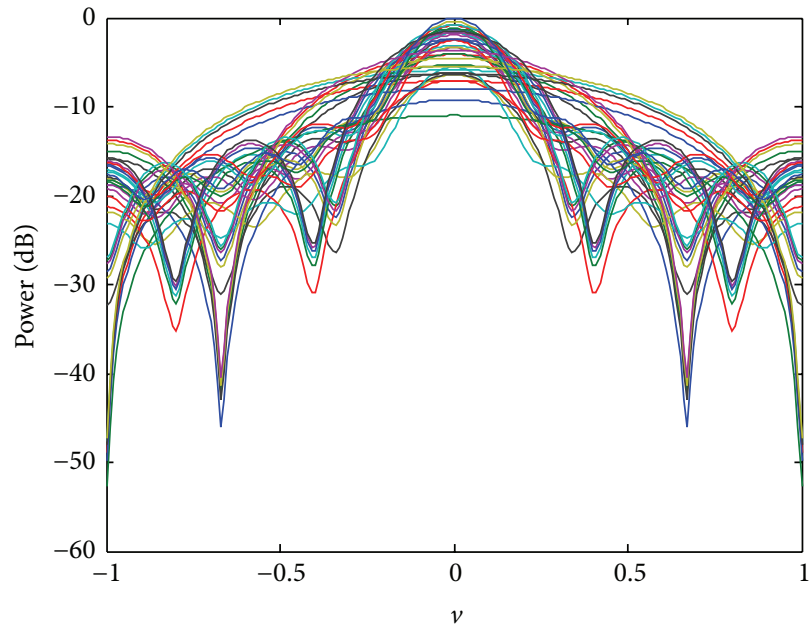

Elevation cut

(a) Original patterns

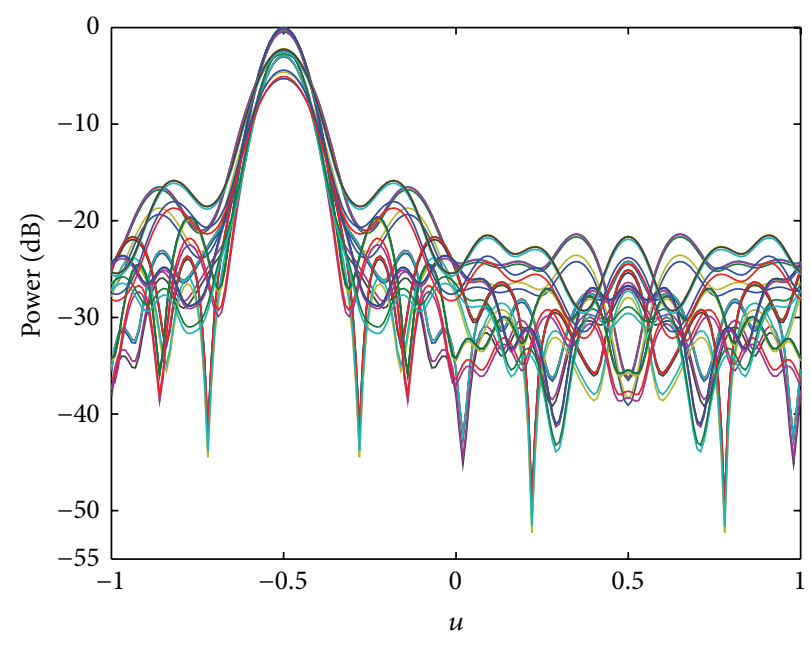

Azimuth cut

(b) GSP

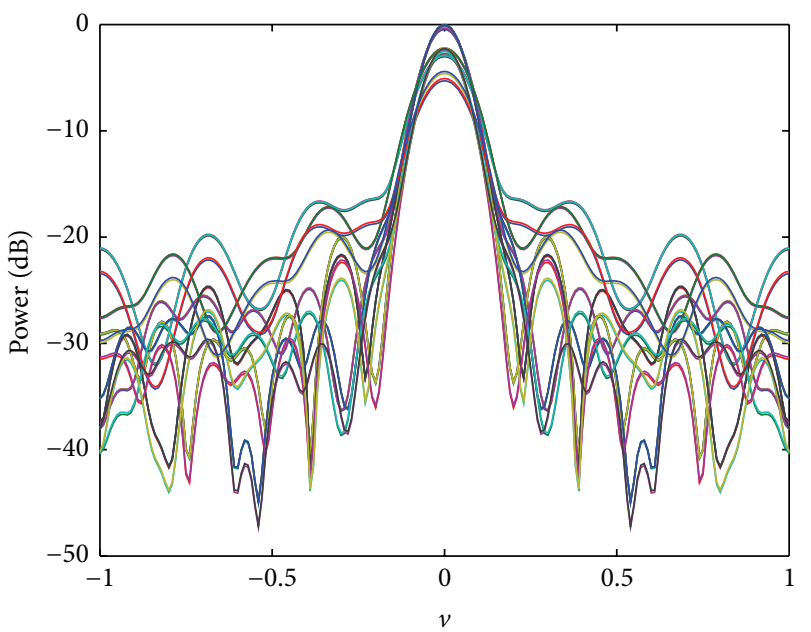

Elevation cut

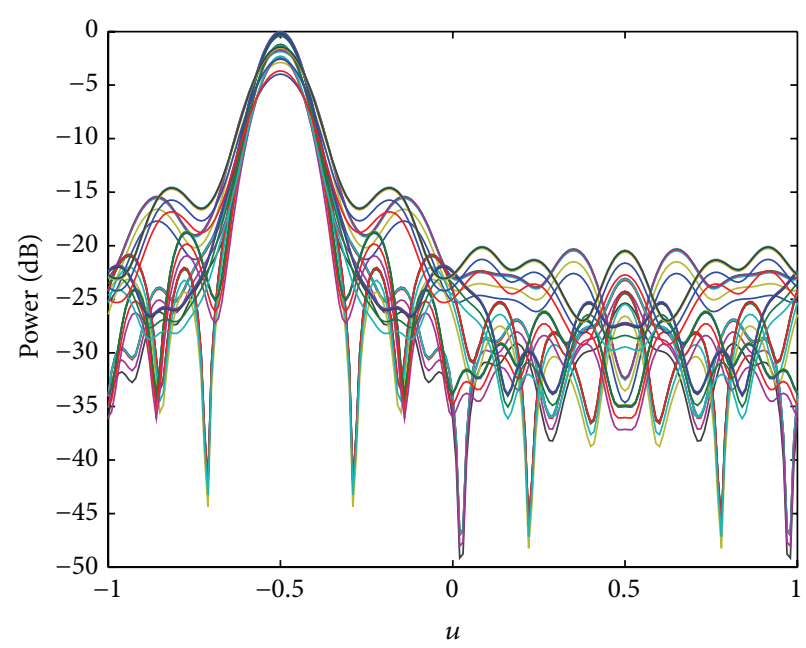

Azimuth cut

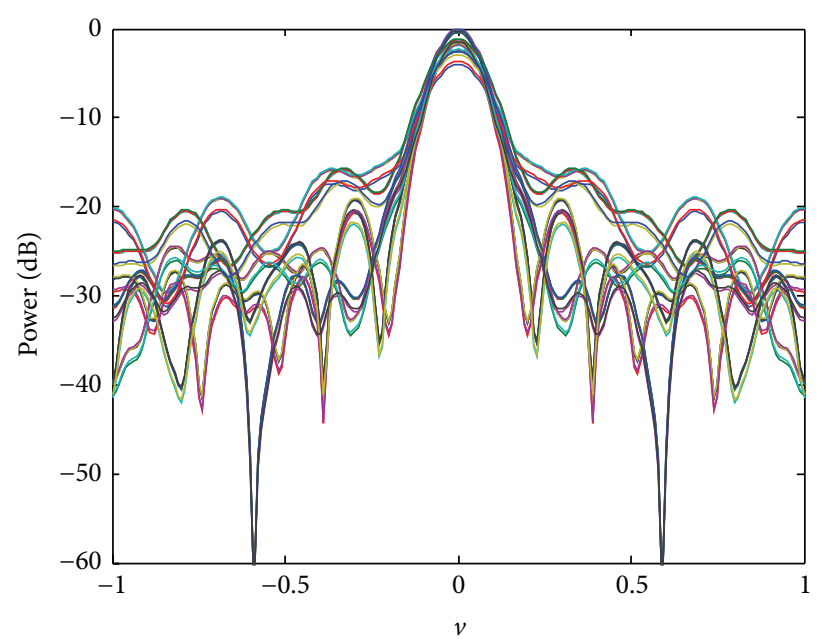

Elevation cut

(c) SGSP

FIGURE 3: Subarray patterns obtained by several methods. 


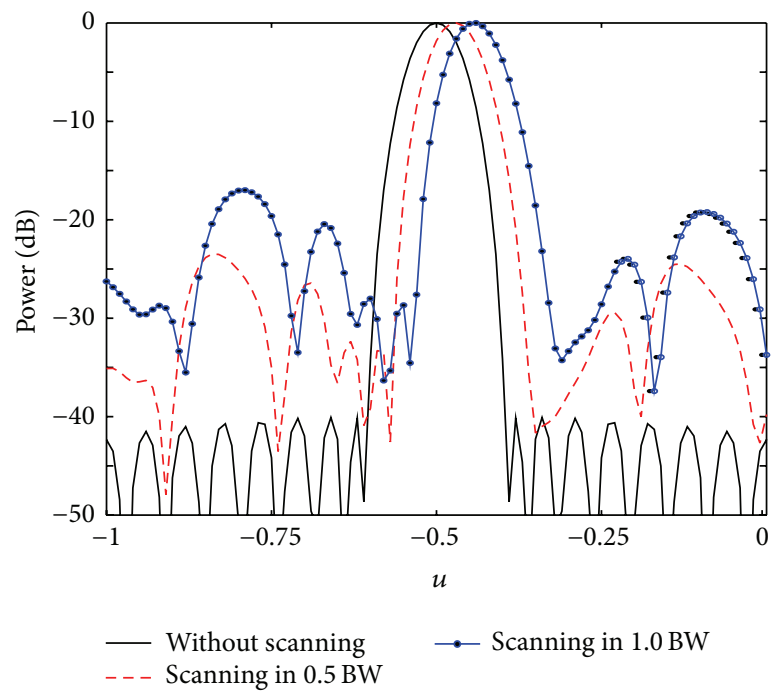

(a) Non-WN

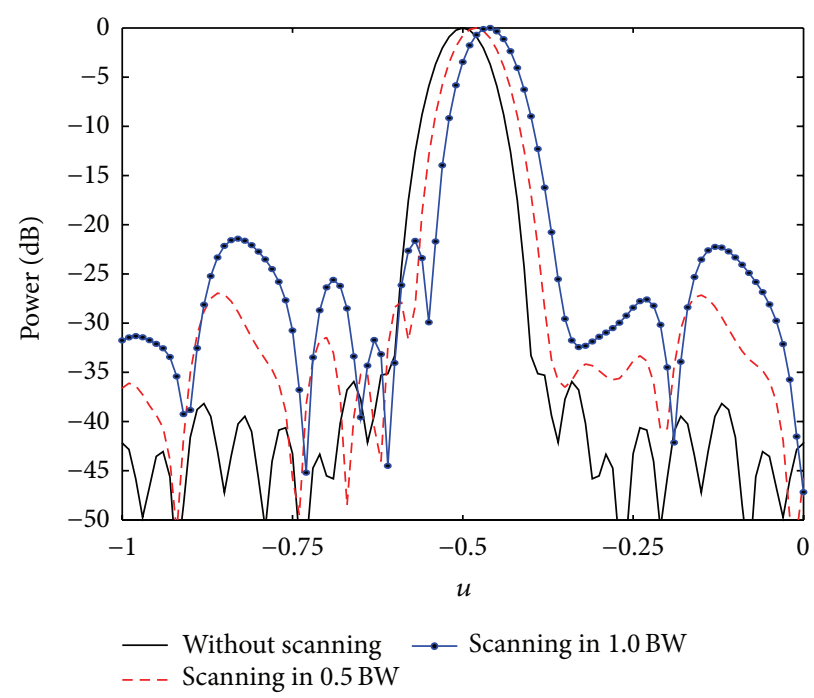

(c) GSP

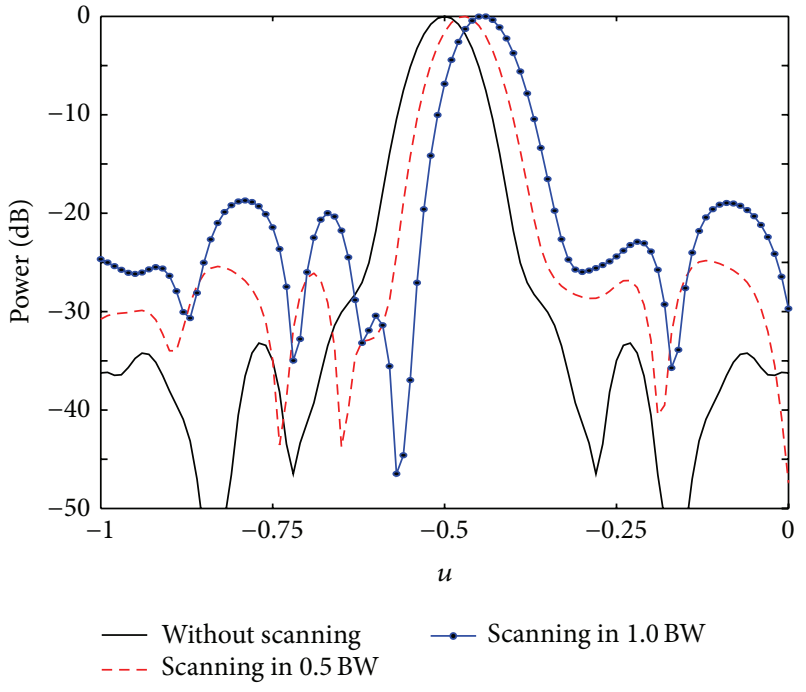

(b) ISDF

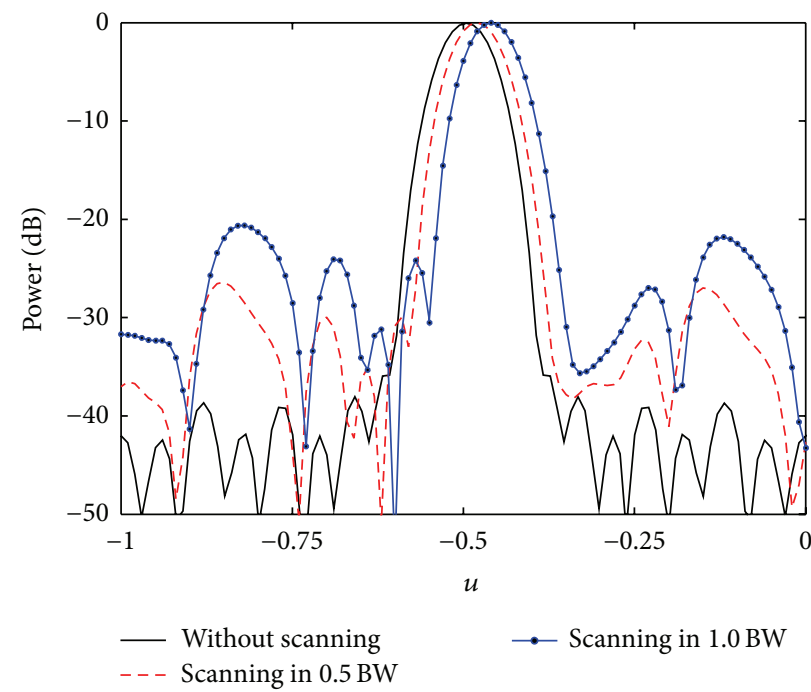

(d) SGSP

FIGURE 4: Array patterns with SBS obtained by different methods (azimuth cut).

subarray patterns. Obviously the shape and the beamwidth of each subarray pattern differ from each other greatly, and the PSL is high. Figure 3(b) shows the subarray patterns obtained by GSP. Obviously, the beam shape and beamwidth of all subarrays are very similar, and the shape of each main beam is similar to Gaussian function while the SLL is reduced effectively. Figure 3(c) shows the subarray patterns obtained by SGSP. We can see that it is very close to Figure 3(b). The beam shape, beamwidth, and gain within the main beam of each subarray are very similar, while the SLL is reduced as well. However, its SLL is slightly higher compared with Figure 3(b).

4.2. The PSL Performance after SBS. Next, simulations after SBS are given, in which scanning is carried out in $u$ direction and the scan angle is set as $0.5 \mathrm{BW}$ and $1.0 \mathrm{BW}$, respectively.
Figure 4 shows the array patterns after scanning. Subplots (a), (b), (c), and (d) show the array patterns obtained without the WN processing, with ISDF, GSP, and SGSP, respectively. The different curves are given for nonscanning and scan angles of $0.5 \mathrm{BW}$ and $1.0 \mathrm{BW}$, respectively. Comparing subplots (c) and (d) with (a), it is obvious that the PSL of the pattern after scanning is reduced using both GSP and SGSP compared to the case without a WN. Comparing subplots (c) and (d) with (b), it is seen that both GSP and SGSP are superior to ISDF in the aspect of the PSL reduction capability. It can be seen that the PSL reduction of SGSP is degraded slightly compared with GSP, which can be seen from subplots (c) and (d).

The PSL values of each pattern in Figure 4 after scanning are shown in Table 1. It can be seen compared to the case without WN that for ISDF the PSL is reduced by $1.3 \mathrm{~dB}$ for 
TABLE 1: The PSL of array pattern with SBS (dB).

\begin{tabular}{lccccccc}
\hline $\begin{array}{l}\text { Scanning range } \\
(B W)\end{array}$ & Non-WN & PSL & ISDF & \multicolumn{2}{c}{ GSP } & \multicolumn{3}{c}{ SGSP } \\
\hline 0.5 & -23.5 & -24.8 & 1.3 & PSL & Improvement & PSL & Improvement \\
\hline 1.0 & -17.0 & -18.7 & 1.7 & -27.0 & 3.5 & -26.4 & 2.9 \\
\hline
\end{tabular}

0.5 BW scanning and $1.7 \mathrm{~dB}$ for $1 \mathrm{BW}$, while for GSP the PSL is reduced by $3.5 \mathrm{~dB}$ and $4.4 \mathrm{~dB}$, respectively. Thus, the PSL of GSP is improved by $2.1 \mathrm{~dB}$ for $0.5 \mathrm{BW}$ scanning and $2.7 \mathrm{~dB}$ for the $1 \mathrm{BW}$ compared with ISDF. Consequently, the GSP improves remarkably ISDF as PSL of the latter is only reduced by more than $1 \mathrm{~dB}$ compared with non-WN. SGSP has a good PSL reduction, too, which PSL is just degraded by $0.6 \mathrm{~dB}$ for $0.5 \mathrm{BW}$ scanning and $0.8 \mathrm{~dB}$ for $1 \mathrm{BW}$, compared with GSP.

\section{Conclusions}

(1) The GSP can reduce the PSL of the array pattern with SBS effectively, while the computational cost is equivalent compared with the ISDF method. For SGSP, the dimension of each matrix for creating the $\mathrm{WN}$ is the same as the subarray number. Consequently, the computational cost is reduced remarkably compared with GSP in which the matrix dimension is equal to the element number. Furthermore, the PSL reduction of SGSP degrades only slightly compared with the latter.

(2) For the WN-based method, the construction of more effective desired subarray pattern is the way to improve the PSL mitigation performance further.

\section{Conflict of Interests}

The authors declare that there is no conflict of interests regarding the publication of this paper.

\section{Acknowledgments}

The authors would like to thank Dr. Ulrich Nickel of Fraunhofer FKIE, Germany, for his insightful comments and useful discussions. This work has been partially supported by the Fundamental Research Funds for the Central Universities (Grant no. HIT.NSRIF.201152), the ASFC (Aeronautical Science Foundation of China, no. 20132077016), and the SAST Foundation (no. SAST201339).

\section{References}

[1] U. Nickel, "Array processing for radar: achievements and challenges," International Journal of Antennas and Propagation, vol. 2013, Article ID 261230, 21 pages, 2013.

[2] A. Farina, G. Golino, and S. Immediata, "Techniques to design sub-arrays for radar antennas," in Proceedings of the International Conference on Antennas and Propagation, pp. 17-23, Exeter, UK, 2003.
[3] P. Lombardo and D. Pastina, "Pattern control for adaptive antenna processing with overlapped sub-arrays," in Proceedings of the International Conference on Radar, pp. 188-193, Adelaide, Australia, 2003.

[4] U. Nickel, "Properties of digital beamforming with subarrays," in Proceedings of the International Conference on Radar (CIE '06), pp. 1-5, Shanghai, China, October 2006.

[5] U. Nickel, "Spotlight MUSIC: super-resolution with subarrays with low calibration effort," IEE Proceedings: Radar, Sonar and Navigation, vol. 149, no. 4, pp. 166-173, 2002.

[6] R. L. Fante, R. M. Davis, and T. P. Guella, "Wideband cancellation of multiple mainbeam jammers," IEEE Transactions on Antennas and Propagation, vol. 44, no. 10, pp. 1402-1413, 1996.

[7] H. Hu and W. H. Liu, "A novel analog and digital weighting approach for sum and difference beam of planar array at subarray level," Chinese Journal of Electronics, vol. 19, no. 3, pp. 468-472, 2010.

[8] L. Manica, P. Rocca, and A. Massa, "Design of subarrayed linear and planar array antennas with SLL control based on an excitation matching approach," IEEE Transactions on Antennas and Propagation, vol. 57, no. 6, pp. 1684-1691, 2009.

[9] Y. L. Wang, K. Q. Duan, and W. C. Xie, "Cross beam STAP for nonstationary clutter suppression in airborne radar," International Journal of Antennas and Propagation, vol. 2013, Article ID 276310, 5 pages, 2013. 

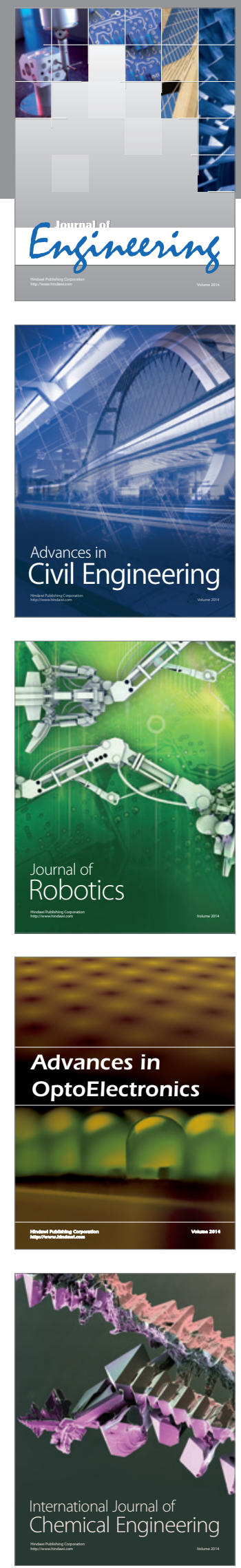

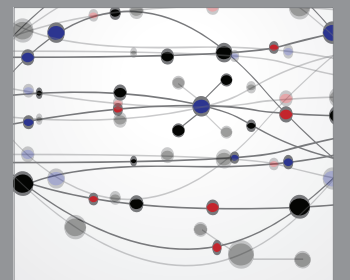

The Scientific World Journal
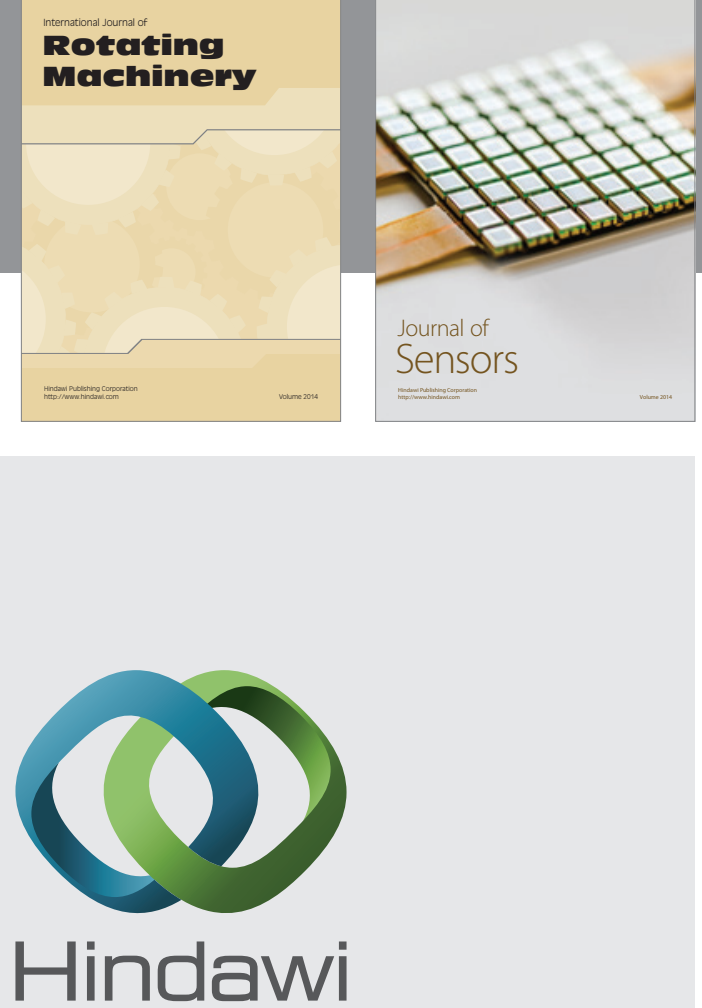

Submit your manuscripts at http://www.hindawi.com
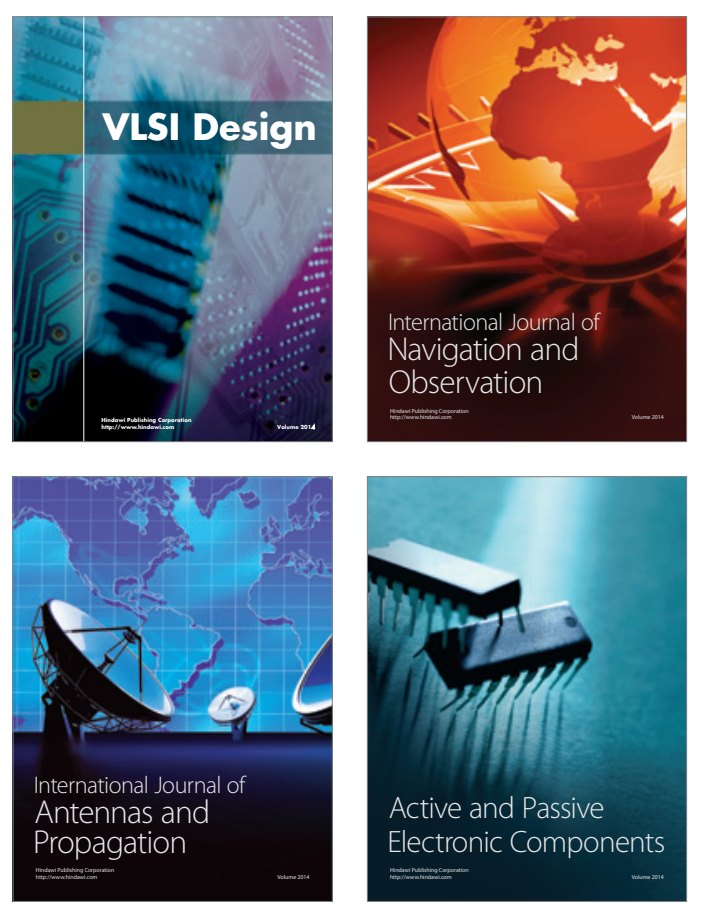
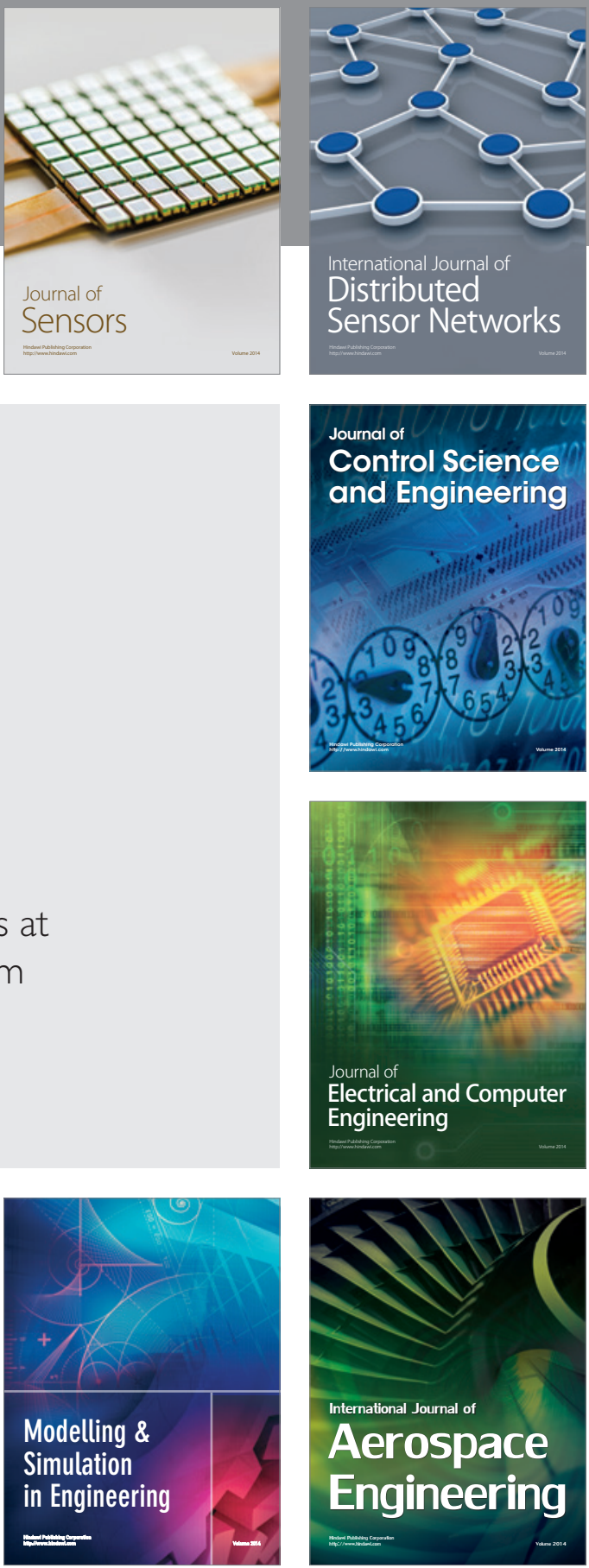

Journal of

Control Science

and Engineering
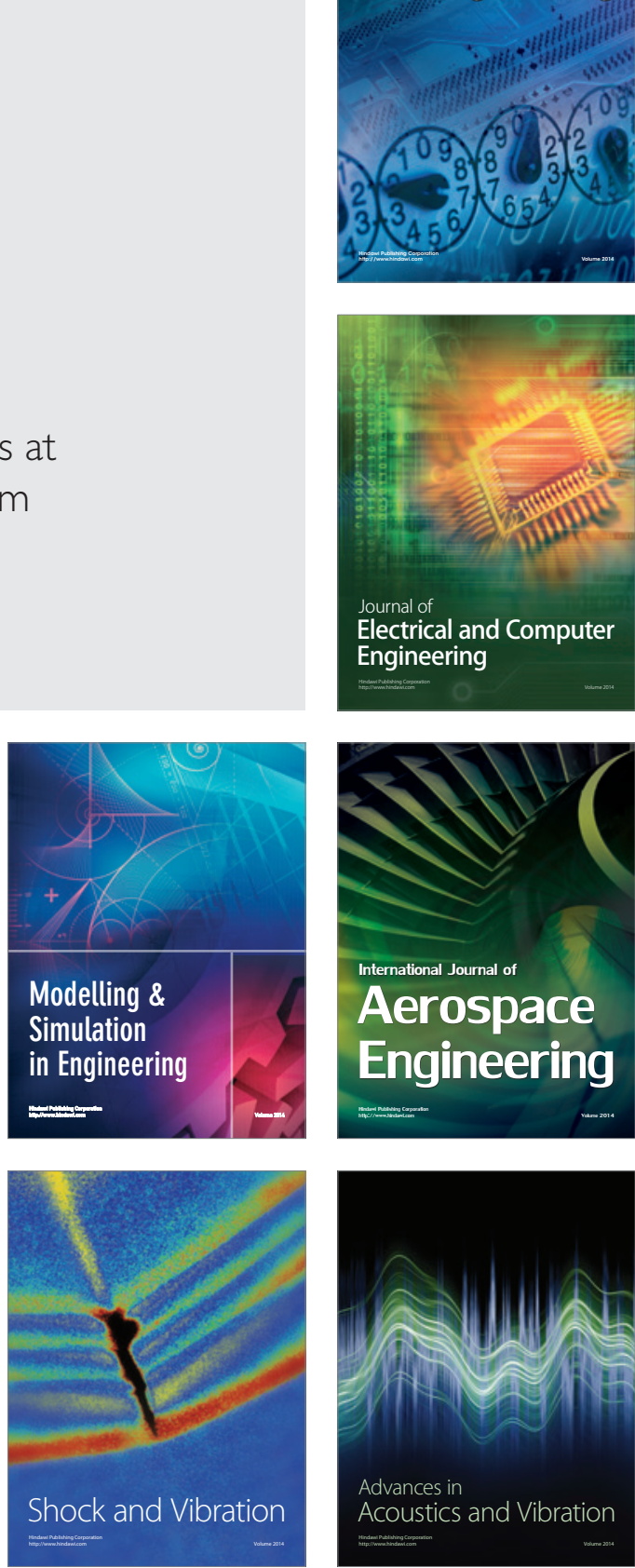